\title{
Grid voltage control with wind turbine inverters by using grid impedance estimation
}

\author{
Jonas De Kooning, Jan Van de Vyver, Jeroen D. M. De Kooning, Tine L. Vandoorn and Lieven Vandevelde \\ Electrical Energy Laboratory (EELAB), Department of Electrical Energy, Systems and Automation (EESA) \\ Ghent University, Sint-Pietersnieuwstraat 41, B-9000 Ghent, Belgium \\ Telephone: +329264 34 22, Fax: +32926435 82, e-mail: Jonas.DeKooning@UGent.be
}

\begin{abstract}
Currently, distributed generation (DG) units usually do not participate in the primary grid voltage control and inject power regardless. Only when the grid voltage level surpasses a certain threshold, these units shut down to avoid any overvoltage problems. In this paper, a method is developed to control the grid voltage by wind turbines. This way, the grid voltage level improves, and the wind turbine can still generate power under suboptimal grid voltage conditions. The effect on the grid voltage depends on the grid impedance ratio $R / X$. In this paper, the effectiveness of the grid voltage control is improved by estimating the grid impedance ratio in real-time to inject active and reactive power accordingly. The method is applied to wind turbines, but can be used for other DG units as well.
\end{abstract}

Keywords-distributed generation, wind turbine inverters, grid voltage control, grid impedance estimation

\section{INTRODUCTION}

A S distributed generation (DG) gains popularity, measures should be taken to make sure these units do not burden the electric power grid [1]. Currently, DG units do not participate in grid voltage control and inject power regardless. On the one hand, if there is a lot of wind and little power consumption, the grid voltage will rise. On the other hand, too much power consumption results in decreased grid voltage levels.

Elevated grid voltage levels can be harmful for the loads connected to the grid. Fortunately, most of these units or the power lines connecting them to the grid - are fitted with overvoltage protections which disconnect them when the grid voltage becomes too high. Either way, overvoltages or disconnection from the grid are both situations to be avoided.

Small wind turbines have an overvoltage protection too, meaning that they shut down when the grid voltage transcends a certain limit. In the Belgian C10/11-standard [2], it is noted that if the distributed generation unit measures a grid voltage deviating $10 \%$ from the nominal value for 10 minutes, it has to shut down. In brief, when there is a lot of wind and little power consumption, either the wind turbine injects all of its available energy and raises the grid voltage unacceptably or all wind energy is wasted due to the disconnection of the wind turbine.

A solution is to control the power output depending on the grid voltage level, [3], [4]. When the grid voltage deviates from the nominal grid voltage, active and reactive power injection is adapted to control the grid voltage accordingly.

It is known that in resistive grids, the grid voltage is mainly influenced by the active power. In inductive grids, the grid voltage is influenced by the reactive power. In hybrid grids however, both active and reactive power have an influence on the grid voltage. In practice, electric grids are always partially resistive and inductive.

Since the grid impedance ratio $R / X$ has an influence on the grid voltage control, a static grid impedance ratio can be hardcoded into the control. However, the active power output could be decreased more than necessary or too much reactive power could be injected, for example. Especially in low-voltage grids, the variance on the grid impedance ratio is large. Furthermore, this ratio changes over time with changing grid load and grid topology.

In this paper, the effectiveness of the grid voltage control is improved by estimating the grid impedance ratio in near realtime to inject active and reactive power accordingly.

The grid voltage control by a wind turbine inverter is derived in section III. This is done by using active and reactive power control, section IV. The grid voltage control is improved by estimating the grid impedance, section $[\mathrm{V}$ The complete control scheme is described in section VI. Finally, the control is tested on a set-up, section VII.

\section{WIND TURBINE SYSTEM}

A small wind turbine system consists of a generator, machineside converter, DC-bus, grid-side converter and filter, as depicted in Fig. 1. The machine-side converter is a passive rectifier with boost chopper which performs a Maximum Power Point Tracking (MPPT) [5]. The power output of this inverter depends on the wind speed and the resulting MPPT. The gridconnected inverter controls the DC-bus voltage by controlling its current output into the grid. This control balances the DCpower being injected into the bus and the AC-power being drawn out of the bus. The active power output of the wind turbine is thus not determined by the grid-connected inverter but by the machine-side converter.

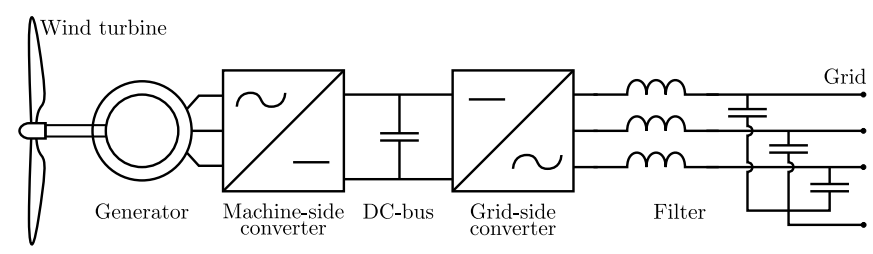

Fig. 1. Wind turbine system 
The grid voltage control explained in this paper is performed by the grid-connected inverter. This converter controls active and reactive power and estimates the grid impedance.

\section{GRID VOLTAGE AND FREQUENCY CONTROL}

As the grid voltage is controlled by active power in resistive grids and by reactive power in inductive grids - and conversely for the grid's frequency, the following droop control equations to control the grid's voltage and frequency are well-known in literature [6]:

$$
\begin{aligned}
& R<<X:\left\{\begin{array}{c}
f-f_{0}=-k_{p}\left(P-P_{0}\right) \\
V-V_{0}=-k_{q}\left(Q-Q_{0}\right)
\end{array}\right. \\
& X<<R:\left\{\begin{array}{c}
f-f_{0}=+k_{q}\left(Q-Q_{0}\right) \\
V-V_{0}=-k_{p}\left(P-P_{0}\right)
\end{array}\right.
\end{aligned}
$$

Equations (1) and (2) represent a droop control since the relation is a proportional controller with droop parameters $k_{p}$ and $k_{q}$. In resistive grids for example, a voltage difference results in a proportional change in the active power output.

The parameters $V$ and $f$ are the instantaneous grid voltage and grid frequency, $P$ and $Q$ are the instantaneous injected active and reactive power by the wind turbine and $R$ and $X$ are the resistance and inductivity of the grid impedance. For voltage and frequency, $V_{0}$ and $f_{0}$ are equal to the nominal grid voltage and frequency respectively. For active and reactive power, $P_{0}$ is equal to the maximum power point (MPP) of the wind turbine and $Q_{0}$ is equal to zero. In the normal situation - when the voltage and frequency equal their nominal values - the active power injection $P$ should be equal to its maximum power point and reactive power injection $Q$ should be zero.

The droop control can be expanded to embed the grid impedance for hybrid grids [6]:

$$
\begin{aligned}
f-f_{0} & =-k_{p} \frac{X}{Z}\left(P-P_{0}\right)+k_{q} \frac{R}{Z}\left(Q-Q_{0}\right) \\
V-V_{0} & =-k_{p} \frac{R}{Z}\left(P-P_{0}\right)-k_{q} \frac{X}{Z}\left(Q-Q_{0}\right)
\end{aligned}
$$

Equations (3) and (4) are rewritten since the grid voltage and frequency are easily measured and the active and reactive powers are easily controlled by the inverter:

$$
\begin{aligned}
P-P_{0} & =-\frac{1}{k_{p}} \frac{X}{Z}\left(f-f_{0}\right)-\frac{1}{k_{p}} \frac{R}{Z}\left(V-V_{0}\right) \\
Q-Q_{0} & =\frac{1}{k_{q}} \frac{R}{Z}\left(f-f_{0}\right)-\frac{1}{k_{q}} \frac{X}{Z}\left(V-V_{0}\right)
\end{aligned}
$$

The result is a droop control for grid voltage and frequency. When the grid voltage deviates from its nominal value, the active and reactive power injection is altered accordingly.

Several remarks and simplifications can be made regarding equations (5) and (6).

1) Neglecting frequency differences: The frequency of the grid is not easily controlled by a DG unit. Unlike the grid voltage which is quite local, the frequency is a global parameter and is primarily controlled by large power plants. In this paper, the wind turbine is a grid-following unit and thus frequency droop control is omitted.
2) Grid impedance ratio $R / X$ : In order to fulfil the active and reactive power control, it is required for the grid impedance $R+j X$ to be known. Although the two variables $R$ and $X$ appear separately in equations (5) and (6), they do not have to be separately known. It is sufficient to know the ratio $R / X$ of the grid impedance. If the grid impedance ratio $\alpha=R / X$ is used, then the following equations arise:

$$
\begin{aligned}
& \frac{X}{Z}=\frac{X}{\sqrt{R^{2}+X^{2}}}=\frac{1}{\sqrt{\left(\frac{R}{X}\right)^{2}+1}}=\frac{1}{\sqrt{\alpha^{2}+1}} \\
& \frac{R}{Z}=\frac{R}{\sqrt{R^{2}+X^{2}}}=\frac{1}{\sqrt{1+\left(\frac{X}{R}\right)^{2}}}=\frac{\alpha}{\sqrt{\alpha^{2}+1}}
\end{aligned}
$$

Substituting (7) and (8) in (5) and (6), together with the omission of frequency droop and a per unit notation, the following active and reactive power droop control results:

$$
\begin{aligned}
& p=-\frac{\alpha}{\sqrt{\alpha^{2}+1}} \frac{1}{k_{p}}\left(v-v_{0}\right)+p_{0} \\
& q=-\frac{1}{\sqrt{\alpha^{2}+1}} \frac{1}{k_{q}}\left(v-v_{0}\right)
\end{aligned}
$$

3) Dependency on time: The parameter $v$ is measured instantaneously at the sample rate of the Analogue-to-Digital Converter (ADC). The maximum power point $p_{0}$ is changed as the wind changes with an order of magnitude of several seconds. The grid impedance ratio $\alpha$ is updated with the frequency of the grid impedance estimation. This is a matter of seconds as well.

\section{ACTIVE AND REACTIVE POWER CONTROL}

In the previous section, the grid voltage droop control was derived. A deviation of the grid voltage from the nominal value results in a certain active and reactive power set point. This section explains how the power output of a wind turbine can be controlled.

The inverter control is executed in the $d q$-reference frame. This frame is synchronised with the measured phase voltage at the point of common coupling (PCC). The result of the Clark/Park transformation is that the $d$-component of the measured voltage is equal to its amplitude and the $q$-component is zero. The rms grid voltage previously noted as $v$, is thus equal to $v_{d}$, and $v_{q}=0$.

\section{A. Reactive power control}

In sinusoidal symmetrical voltage systems, the expression for reactive power is equal to:

$$
q(t)=-\frac{3}{2} v_{d}(t) i_{q}(t)
$$

Substituting equation 10 in equation 11 gives:

$$
i_{q}(t)=\frac{2}{3} \frac{1}{\sqrt{\alpha(t)^{2}+1}} \frac{1}{k_{q}}\left(1-\frac{v_{0}}{v_{d}(t)}\right)
$$

Reactive power can be controlled by adapting the set value of the $q$-component current. A deviation of the grid voltage from the nominal value results in a set value for the $q$-component current through the droop control of equation (12). 
The grid-connected inverter can inject and absorb reactive power. This means that the grid voltage can be controlled both up and down by using reactive power control.

The control of the $q$-component current is basically controlling the phase difference between output voltage and current. This is why reactive power can be controlled instantaneously.

\section{B. Active power control}

Since the grid-connected inverter controls the balance of DCand AC-power of the bus, the active power output of the gridconnected inverter will be equal to the output of the machineside converter. The machine-side converter performs an MPPT such that its active power output will be $P_{0}$. However, the MPPT control can be adapted to control the active power output below the MPP. The result is that the active power output of the grid-connected inverter can be controlled through adapting the active power output of the machine-side converter.

The instantaneous maximum power point $p_{0}$ can be communicated with the grid-connected inverter since it is required for the active power droop control in equation (9). With the notation of time:

$$
p(t)=-\frac{\alpha(t)}{\sqrt{\alpha(t)^{2}+1}} \frac{1}{k_{p}}\left(v_{d}(t)-v_{0}\right)+p_{0}(t)
$$

Active power can be controlled by adapting the set value of the power point $p$. A deviation of the grid voltage from the nominal value results in a set value for active power $p$ through the droop control of equation (13).

Since the maximum power point $p_{0}$ is actually the maximum possible active power output, only overvoltages can be corrected since only a decrease in active power can be controlled. For an undervoltage in the grid, droop control equation (13) dictates a power output $p>p_{0}$. Since $p_{0}$ is determined by the MPPT, $p$ cannot be larger than $p_{0}$ and saturates on this value.

The issue of undervoltages was already present in times when DG units were not yet established. With the rising influence of DG units, typically overvoltages occur in the grid. This is why overvoltages are more important than undervoltages and active power control is an important factor in the grid voltage control. Also, undervoltages can be controlled by the reactive power control.

Changing the active power output of the machine-side converter requires time. The generator and wind turbine have to reach a steady-state speed when changing the set point of power. This can be a matter of seconds. This is why active power is not controlled instantly but has a certain delay.

\section{GRID IMPEDANCE ESTIMATION}

In the grid voltage droop control, the grid impedance ratio $R / X$ appears. This value is used to weigh the amount of active and reactive power control used for controlling the grid voltage. In high-voltage transmission grids, this value is around 0.3 . In medium-voltage grids, it is around 0.8. In low-voltage distribution grids, the grid impedance ratio can vary between 2 and 8.

Numerous methods for estimating the grid impedance exist. These methods can be differentiated based on whether they estimate while receiving new measurements (online) or have to store all the samples first (offline), if they deliberately disturb the grid with a signal (active) or only observe the grid (passive), and if they measure transient effects (transient) or steady-state phenomena (steady-state).

One passive method uses an Extended Kalman Filter (EKF) [7], but this method is bound to be computationally too heavy. Another uses active filtering of an LCL-filter [8]. This method is only capable of estimating the inductivity of the grid impedance and requires additional measurements of the current.

The method which is the easiest in practical application and does not require additional measurements or components, is the non-characteristic current injection [9]. In this method, a harmonic current is injected into the grid, a discrete Fourier transform (DFT) of both voltage and current is calculated and the estimated grid impedance results. This method is discussed in the remaining of this section and will be used for the grid voltage control.

\section{A. Principle of operation}

The goal is to obtain the impedance at grid frequency, which is $50 \mathrm{~Hz}$ in European grids. By injecting a harmonic current and measuring the voltage response, it is possible to obtain the grid impedance at the grid frequency.

For every harmonic frequency, a Thévenin equivalent can be calculated of the grid impedance and source. The result is the following impedance for harmonic order $h_{i}$ :

$$
\vec{Z}_{g}\left(h_{i}\right)=R_{g}\left(h_{i}\right)+j X_{g}\left(h_{i}\right)
$$

The impedance can be calculated as:

$$
\frac{\vec{V}_{g}\left(h_{i}\right)}{\vec{I}_{g}\left(h_{i}\right)}=\vec{Z}_{g}\left(h_{i}\right)
$$

For a certain harmonic, the calculated grid impedance is not equal to the one at grid frequency:

$$
\vec{Z}_{g}(50 \mathrm{~Hz}) \neq \vec{Z}_{g}\left(h_{i}\right)
$$

Writing the inductive component as $X_{g}\left(h_{i}\right)=\omega\left(h_{i}\right) L_{g}\left(h_{i}\right)$, the parameters $R_{g}\left(h_{i}\right)$ and $L_{g}\left(h_{i}\right)$ depend on the harmonic order due to the skin- and proximity effect. For a harmonic close to the grid frequency, these have little effect. The grid's rotational frequency $\omega=2 \pi 50 \mathrm{rad} / \mathrm{s}$ differs from the one at harmonic frequency.

To estimate the grid impedance by using a harmonic $h_{i}$, the following is calculated:

$$
\vec{Z}_{g}(50 \mathrm{~Hz}) \simeq \Re\left[\frac{\vec{V}_{g}\left(h_{i}\right)}{\vec{I}_{g}\left(h_{i}\right)}\right]+j \frac{2 \pi 50 \mathrm{rad} / \mathrm{s}}{\omega\left(h_{i}\right)} \Im\left[\frac{\vec{V}_{g}\left(h_{i}\right)}{\vec{I}_{g}\left(h_{i}\right)}\right]
$$

The values $\vec{V}_{g}\left(h_{i}\right)$ and $\vec{I}_{g}\left(h_{i}\right)$ are calculated by using a DFT of the voltage and current during the injection of the harmonic current.

The harmonic of the injected current should be close to the fundamental grid frequency to justify the above approximations. Also, the harmonic may not be significantly present in the grid. As suggested in [9], a $75 \mathrm{~Hz}$ harmonic is used as harmonic injected current. 


\section{B. Harmonic current injection}

Since the inverter control is executed in the $d q$-reference frame, the set values of the $d$ - and $q$-component current should be appropriately controlled to result a $75 \mathrm{~Hz}$ harmonic current in the output in the $a b c$-reference frame.

It can be derived that in order to obtain a $75 \mathrm{~Hz}$ harmonic current, the set values have to be:

$$
\begin{aligned}
& i_{d, \mathrm{set}}=i_{d, \mathrm{set}, 50 \mathrm{~Hz}}-B \cos (2 \pi 25 t) \\
& i_{q, \mathrm{set}}=i_{d, \mathrm{set}, 50 \mathrm{~Hz}}+B \sin (2 \pi 25 t)
\end{aligned}
$$

where $i_{d q, \text { set, } 50 \mathrm{~Hz}}$ are the current set values posed by the bus voltage controller and reactive power controller. The factor $B$ is the amplitude of the injected current. This value depends on the rated power of the wind turbine and is a consideration between a high signal-to-noise ratio and a low total harmonic distortion (THD). A proper magnitude of $B$ is determined experimentally in section $\mathrm{VII}$.

\section{Running-sum DFT}

To calculate the required $\vec{V}_{g}\left(h_{i}\right)$ and $\vec{I}_{g}\left(h_{i}\right)$, a DFT of the samples of the voltage and current has to be calculated.

A first issue is how long the harmonic current has to be injected and how many samples have to be used for the DFT. Since the injected harmonic current increases the THD, the process should be as short as possible. For a proper DFTcalculation, the process should be long enough to receive enough information to extract the harmonic. In [9], it is suggested to inject for $40 \mathrm{~ms}$. This way, exactly two periods of the grid waveforms and three periods of the harmonic current will appear. This is the shortest period wherein both the grid waveforms and the harmonic appear as a integer amount of periods. The fundamental frequency of the DFT equals $f_{s} / N=25 \mathrm{~Hz}$, with $f_{s}$ the sampling frequency and $N$ the number of samples. This way, the third harmonic will be the $75 \mathrm{~Hz}$ harmonic.

A second issue is the required memory space. For a DFT sampling at $3 \mathrm{kHz}$, a period of $40 \mathrm{~ms}$ will require 120 samples. By using a running-sum implementation of the DFT, less memory space is required.

The expression for the DFT of the third harmonic $(75 \mathrm{~Hz})$ is as follows:

$$
\vec{V}(3)=\frac{1}{N} \sum_{n=0}^{N-1} v(n) e^{-j \frac{2 \pi}{N} 3 n}
$$

with $N=2 / 50 f_{s}$ the number of samples and $v(n)$ the sampled source waveform.

\section{Simulations}

The grid impedance estimation method is simulated in a Matlab Simulink model. This model consists of a three-phase source, resistors and inductors as line impedances, a load, three-phase inverter and a DC-source. The scheme of the model is depicted in Fig. 2.

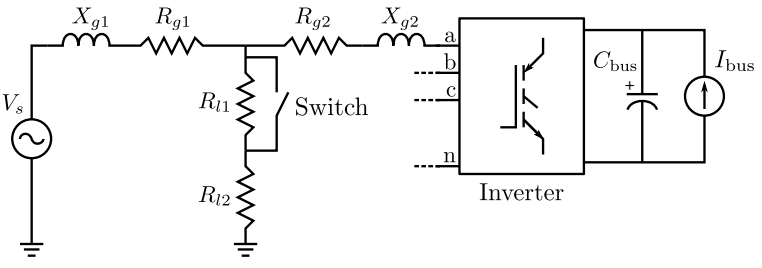

Fig. 2. Scheme of the simulation model containing the DC-source, inverter, grid model and grid source. The depicted scheme represents one phase of the three-phase set-up.

The resulting estimate is compared to the impedance used in the grid model, see Fig. 3

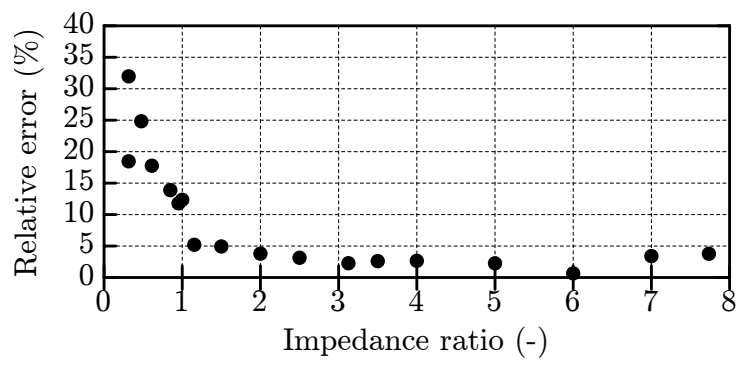

Fig. 3. Relative error on $\alpha$ for simulation of the grid impedance estimation.

The grid impedance is estimated with a low error for impedance ratios above unity. Below unity however, the error increases rapidly. Further investigation showed that this is caused by an error on the resistive component of the grid impedance, see Fig. 4 The inductive component retains a significant low error for the whole simulated interval of impedance ratios. The reason is that the grid voltage gets disturbed increasingly for inductive grids and this affects the DFT.

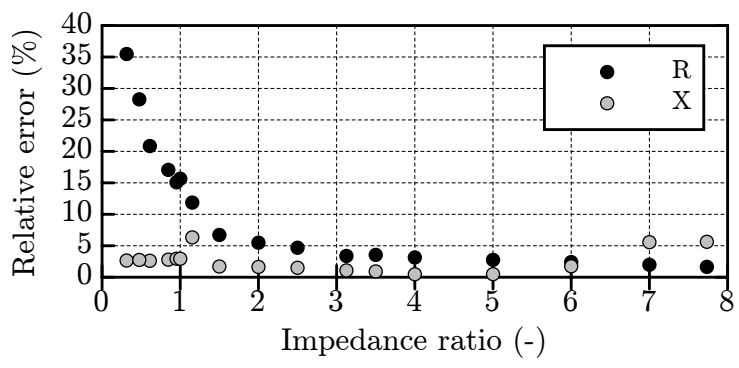

Fig. 4. Relative error on the $R$ - and $X$-component for simulation of the grid impedance estimation.

\section{CONTROL SCHEME}

The grid voltage control by using active and reactive power and grid impedance estimation is depicted in Fig. 5

The measured grid voltage is written as $v_{g}$ and the measured line current as $i_{L}$. The active and reactive power control blocks embed equations (12) and (13). The grid impedance estimation block embeds equation (17). The block GIE-control - where GIE stands for Grid Impedance Estimation - controls when a new estimation of the $R / X$ is required and enables both the harmonic current injection and DFT-calculation for a duration of $40 \mathrm{~ms}$. 


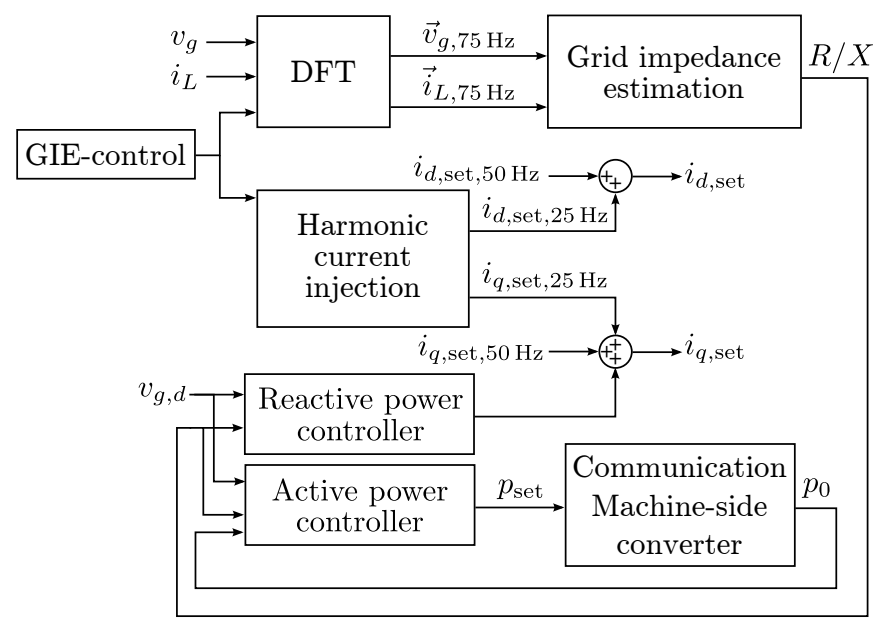

Fig. 5. Scheme of the grid voltage control by using active and reactive power and grid impedance estimation

\section{EXPERIMENTS}

The grid voltage control by using active and reactive power and grid impedance estimation is tested on a practical set-up. The set-up is depicted in Fig. 2 The resistors and inductors are adjustable to test various grid impedances and a switch is used to test a sudden load change.

\section{A. Grid impedance estimation}

Measurements are performed to test the grid impedance estimation. The grid impedance is adjusted with every measurement to change the grid impedance ratio. The measurement is compared with the analytically calculated impedance. For each impedance ratio, 400 samples of the grid impedance are saved over a period of one minute. The amplitude of the injected harmonic equals $0.4 \mathrm{~A}$ superposed on currents with an rmsvalue of $1.66 \mathrm{~A}$. The medians of these samples are depicted in Fig. 6

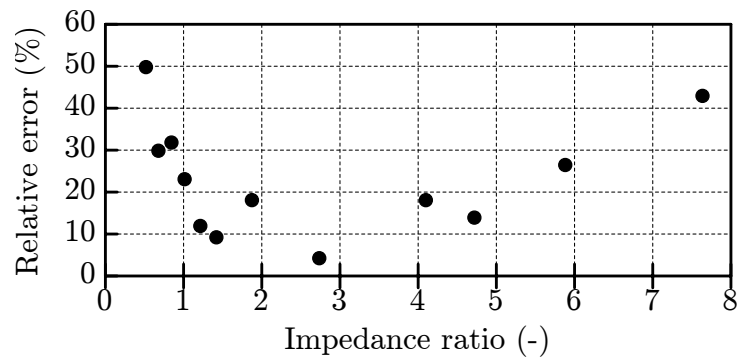

Fig. 6. Measured relative error of the median of 400 samples compared to the analytical grid impedance ratio $R / X$.

As the simulations have shown, the relative error increases for low impedance ratios. However, the error increases for increased impedance ratios as well. To increase the accuracy at higher impedance ratios, the amplitude of the injected harmonic current has to be increased, see Fig. 7] where at a grid impedance ratio of 7.64 various current amplitudes are tested.

An error on the grid impedance estimation influences the grid voltage control. It is important to notice that the measured

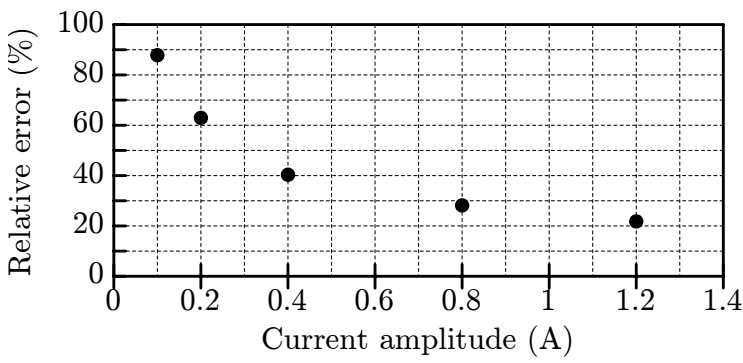

Fig. 7. Measurement of the relative error of the grid impedance estimation for various injected harmonic current amplitudes for a grid impedance ratio of 7.64 .

relative error of the grid impedance ratio is always an overestimation. This means that the estimated result indicates a higher resistive component than actually present in the grid. When looking at the grid voltage control, this would mean that more active power - and less reactive power - is used in controlling the voltage than required, which is undesired.

A solution is to calibrate the grid impedance estimator to reduce the relative error. Another is to increase the amplitude of the injected harmonic current. But this only has significant effect on larger impedance ratios. A third is to change the weighting factors for active and reactive power control - as written in equations (7) and (8) - to linear functions:

$$
\begin{aligned}
& \frac{1}{\sqrt{\alpha^{2}+1}} \longrightarrow 1-\frac{\alpha}{8} \\
& \frac{\alpha}{\sqrt{\alpha^{2}+1}} \longrightarrow \frac{\alpha}{8}
\end{aligned}
$$

The parameter $\alpha$ is normalised on 8 since this is the maximum expected grid impedance ratio. Because the used factors of (7) and (8) change a lot for low grid impedance ratios, an error on the estimate has a larger effect. With the linear functions in (7) and (8), this is not the case.

\section{B. Grid voltage control}

By using the practical set-up, measurements of the grid voltage control are performed for various grid impedance ratios. The grid impedance is estimated by using the method described in section $\mathrm{V}$ and tested in VII-A. For each ratio, the grid voltage is measured for two cases: without and with voltage control.

The first case is measured when the wind turbine injects all of its available power by MPPT. The second when the grid voltage is controlled by using the method presented in this paper. The nominal voltage used as set value for the grid voltage control is depicted as well.

Measurements where an overvoltage occurs, are depicted in Fig. 8 It can be noticed that the improvement in grid voltage - thus a decrease - is greater for low grid impedance ratios, thus for inductive grids. For higher impedance ratios, the grid voltage improvement is less.

An important reason why the grid voltage control appears to be performing less in resistive grids, can be found in Fig. 8 as well. The voltage level when the inverter injects all of its available power - denoted 'No voltage control' in the figure, is lower for higher impedance ratios in comparison to lower impedance ratios. This is caused by the grid model set-up 


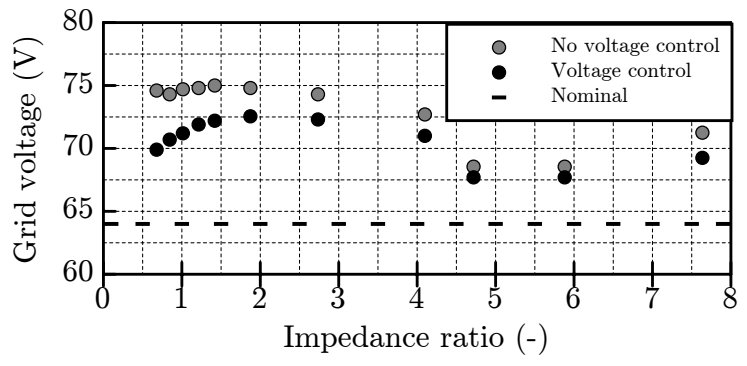

Fig. 8. Grid voltage control for various grid impedance ratios.

not being symmetrical: $\vec{Z}_{g 1} \neq \vec{Z}_{g 2}$, as shown in Figure 2 The inductance and resistance values between the inverter and the load, and the load and the source are not identical. The impedance between the inverter and the load causes a larger voltage drop for higher impedance ratios. Because of this, the voltage difference with the nominal set value of the grid voltage is smaller. Since this voltage difference is used as input for both the active and reactive power controller, these controllers respond with less decrease in DC-current and increase in $q$-component current respectively. The result is that the grid voltage is controlled less.

When looking at the improvement in grid voltage difference on a relative basis, the improvement is about $20 \%$ for grid impedance ratios above 1.5 . Below this value, the improvement raises up to $40 \%$.

The DC-current output of the machine-side converter is controlled to control the active power. In the experiments, this is done by controlling the current output of a DC-current source. As seen in Fig. 9, the active power is decreased for a larger voltage difference.

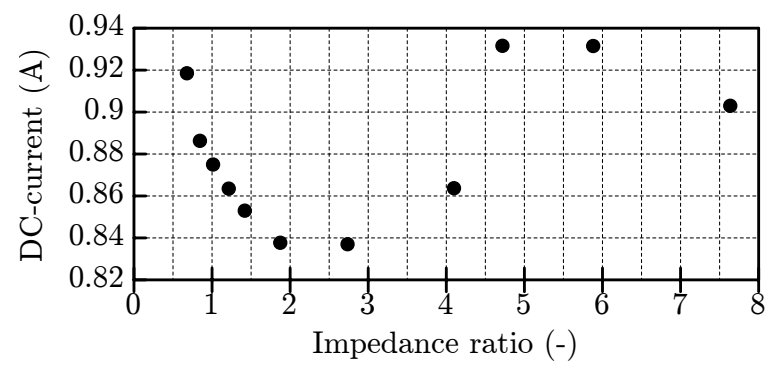

Fig. 9. Controlled DC-current of the machine-side converter.

\section{CONCLUSIONS}

The grid voltage control as investigated in this paper, is confirmed - by using a practical set-up and measurements - to improve the grid voltage and avoid renewable energy sources to shut down when the voltage increases too much. By reducing the active power output of a renewable energy source, a part of the full potential of energy production is sacrificed to gain a grid voltage level closer to its nominal value. This sacrifice is for the better: the alternative is to completely shut down the energy source wherein the full potential of energy production is sacrificed.

The effectiveness of the grid voltage control is improved by estimating the grid impedance ratio. This way, the grid voltage control can be applied for wind turbines connected to any type of grid and it responds on changes in grid load and topology.

The benefit of grid voltage control by wind turbine inverters is twofold. Because the energy source does not completely shut down in the case of an overvoltage - but reduces its power output, the total generated energy of a particular wind turbine on a yearly basis will increase. Also, the grid voltage levels are improved because these are controlled by the wind turbine inverter.

\section{REFERENCES}

[1] A. Keane, L. F. Ochoa,C. L.T.Borges, G. W. Ault, A. D. AlarconRodriguez, R. A. F. Currie, F. Pilo, C. Dent and G. P. Harrison, "Stateof-the-Art Techniques and Challenges Ahead for Distributed Generation Planning and Optimization," IEEE Trans. on Power Systems, vol. 28, pp. 1493 - 1502, May 2013.

[2] Synergrid, C10/11 standard - Specific technical prescriptions for distributed generation units operating in parallel in the distribution grid, June 2012.

[3] J. C. Vasquez, J. M. Guerrero, A. Luna, P. Rodríguez and R. Teodorescu, "Adaptive Droop Control Applied to Voltage-Source Inverters Operating in Grid-Connected and Islanded Modes," IEEE Trans. on Industrial Electronics, vol. 56, pp. 4088-4096, October 2009.

[4] F. Wang, J. L. Duarte and M. A. M. Hendrix, "Pliant Active and Reactive Power Control for Grid-Interactive Converters Under Unbalanced Voltage Dips," IEEE Trans. on Power Electronics, vol. 26, pp. 1511-1521, May 2011.

[5] E. Koutroulis and K. Kalaitzakis, "Design of a Maximum Power Tracking System for Wind-Energy-Conversion Applications," IEEE Trans. on Industrial Electronics, vol. 53, pp. 486-494, April 2006.

[6] K. De Brabandere, B. Bolsens, J. Van den Keybus, A. Woyte, J. Driesen and R. Belmans, "A Voltage and Frequency Droop Control Method for Parallel Inverters," IEEE Trans. on Power Electronics, vol. 22, pp. 11071115, July 2007.

[7] N. Hoffmann and F. W. Fuchs, "Online Grid Impedance Estimation for the Control of Grid Connected Converters in Inductive-Resistive Distributed Power-Networks Using Extended Kalman-Filter," in Energy Conversion Congress and Exposition (ECCE), 2012 IEEE, pp. 922-929, September 2012.

[8] M. Liserre, F. Blaabjerg and R. Teodorescu, "Grid Impedance Estimation via Excitation of LCL-Filter Resonance," IEEE Trans. on Industry Applications, vol. 43, pp. 1401-1407, September 2007.

[9] L. Asiminoaei, R. Teodorescu, F. Blaabjerg and U. Borup, "Implementation and Test of an Online Embedded Grid Impedance Estimation Technique for PV Inverters," IEEE Trans. on Industrial Electronics, vol. 52, pp. 1136-1144, August 2005. 